\title{
Eye-safe Laser Line Striper for Outside Use
}

\author{
C. Mertz, J. Kozar, J.R. Miller, C. Thorpe \\ Robotics Institute \\ Carnegie Mellon University \\ Pittsburgh, PA 15213-3890 \\ Tel.: 01-412-2683445 \\ Fax:01-412-2687350 \\ cmertz@andrew.cmu.edu
}

\begin{abstract}
Collision warning or autonomous driving in cluttered environments like urban areas require short-range high-resolution sensors. Range finders using laser triangulation fulfils these requirements but they face the additional challenge of operating in bright sunlight while remaining eye-safe. The laser line striper introduced in this paper achieves all these requirements without the need of expensive components. The sensor can be built with a variety of field-of-views.
\end{abstract}

Keywords: range finder, laser triangulation, eye safety, image sensor, sensing in sunlight, structured light, infrared laser

\section{Introduction}

It is an obvious goal for a vehicle manufacturer to make the vehicle safe and convenient. To avoid dangerous situations, more and more vehicles are equipped with warning systems which alert the driver. Some systems go a step further and take control of the vehicle, notably adaptive cruise control. These systems deal mostly with situations on highways, where detected objects are large and relatively well defined (other vehicles) and at medium and large distances. In stark contrast is the operational environment of urban areas, which consists of small and large fixed objects, such as other vehicles, pedestrian, bicyclists, etc. up to very close distances. A warning system does not only need to find these targets to alert the driver about a possible collision, it also should have some understanding of the environment [1] to cut down on unnecessary alarms. Of particular interest in one of our research projects [2] is the detection of the curb. Pedestrian can sometimes be very close to the vehicle, but the driver does not need to be warned, as long as the pedestrian is on the curb.

It is desirable to have a sensor which is able to:

1. Work outside under most weather conditions, specifically bright sunlight.

2. Be eye-safe.

3. Measure a large range of angles.

4. Range of 0.5-4 meters.
5. High resolution (few $\%$ in distance, $<1^{\circ}$ in angle).

6. Low cost (in the order of $\$ 100$ mass produced).

7. Small size (mountable in vehicles).

8. Robust.

9. Acquisition rate of at least $20 \mathrm{~Hz}$.

10. Detect fast moving objects (up to $100 \mathrm{~km} / \mathrm{h}$ )

\section{Line Striper}

To achieve high angular resolution while keeping the size of the sensor small, one needs to make use of radiation with small wavelengths, i.e. one needs to use visible or IR light. Measurement of a large range of angles requires the sensor either to consist of many sub-sensors or to make very rapid sequential measurements. Distance can be measured either by time-of-flight (TOF) or triangulation. Our choice is a triangulating system consisting of a camera and a laser. Such a system is less expensive than TOF systems and, not having any moving parts, is less susceptible to mechanical failure.

Active triangulation has been used in many different ways before [3-6], but none of these sensors fulfil all 10 criteria mentioned above.

\section{Active triangulation}

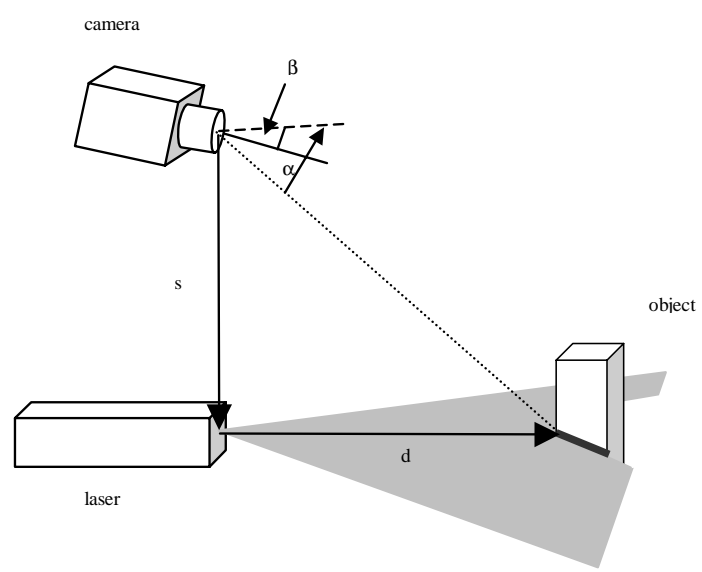

Figure 1 Geometric configuration of the laser and camera. 
The focused light of a laser is passed through a cylindrical lens which strongly defocuses the beam in one direction and leaves the beam unaffected in the other direction. It is thereby possible to create a light plane which projects lines on intersecting objects (see Figure 1). A camera at the distance $s$ from the laser sees this line as a set of pixels, which each correspond to angles $\alpha$ and $\beta$. The distance $d$ can be determined from the angle $\alpha$ :

$$
d=s \cdot \cot (\alpha)
$$

\section{From image to distance}

The raw data received are the pixel intensity values in image, representing the light intensity of the scene. The first step is to identify which pixels correspond to the line projected by the laser (e.g. Figure 3 and top of Figure 5). In the absence of background, this could simply be done by applying a threshold above the noise level. In a configuration where the centre of the camera is directly above the origin of the laser (as shown in Figure 1) there is a one-to-one correspondence between the columns and $\beta$. There is only one position in each column which corresponds to the line. Therefore we identify the line as the pixels with maximum intensity in each column above a preset threshold.

To get from the pixels to the external coordinates we determine the internal parameters of the camera [8] and thereby the undistorted image coordinates. Next, the geometric relationship between the camera and the laser plane is being measured. Now it is possible to calculate the homology between the image plane and the laser plane.

To increase the resolution of the system we also implemented sub-pixel localisation of the line. The new location is the intensity weighted average of the previously determined pixel and its two nearest neighbours in the same column. In the ideal case of no background, strong signal, and uniform albedo of the object, we were able to improve the resolution by a factor of about 10 . It has been reported, that one can achieve an improvement by a factor of about 50 if the detailed shape of the laser beam is taken into account [9]. The final distance measurements from the above mentioned scenes can be seen in Figure 4 and bottom of Figure 5.

\section{Choice of wavelengths}

To avoid the maximum intensity of the sun around one can use UV or IR light sources. UV lasers are more hazardous and much more expensive than IR, so IR is the natural choice. The longer the IR wavelength is, the less dangerous is the light to the eye, since it is less focused on the retina. On the minus side, inexpensive cameras have reduced sensitivity for longer IR wavelengths. We selected GaAs laser diodes, which are relatively inexpensive and have a wavelength of around $900 \mathrm{~nm}$, a good compromise between reduced sensitivity of the camera and increased eye safety.

\section{Eye safety}

Since the sensor is expected to be operated close to humans, the incorporated laser should be class I. The criteria for laser safety can be found in Ref. [7]. As is discussed later, we will be using laser pulses of around $1 \mu$ s length at a repetition rate of $30 \mathrm{~Hz}$. As an example the laser projects a line which is fanned out to $60^{\circ}$ in one direction and to a thickness of less than $0.7 \mathrm{~cm}$ in the other, and the laser will be in an enclosure so that one can not get closer than $60 \mathrm{~cm}$ to the laser. The maximum permissible energy per pulse is then:

$E=M P E \cdot A \cdot \frac{\pi \cdot 60 \mathrm{~cm}}{3 \cdot 0.7 \mathrm{~cm}}$

$A=0.385 \mathrm{~cm}^{2}$ area of pupil

$M P E=0.5 \cdot C_{p} \cdot C_{A} \cdot 10^{-6} \frac{\mathrm{J}}{\mathrm{cm}^{2}}$

$C_{A}=10^{2(\lambda-0.700)}$

$\lambda=0.900 \quad$ (wavelength )

$C_{p}=(10 \mathrm{~s} \cdot 30 \mathrm{~Hz})^{-0.25}$

$\Rightarrow E=10.4 \mu \mathrm{J}$

This is equivalent to about $0.3 \mathrm{~mW}$ of average power.

\section{Background suppression}

The laser emits a line of $60^{\circ}$ length and $0.4^{\circ}$ thickness, giving an average laser light at distance $2 \mathrm{~m} 8 \times 10^{4}$ times less intense than sunlight. There are three main ways to suppress the large background of the sun:

1. pulsed laser and fast camera shutter

2. narrow filter

3. background subtraction

We have implemented the first two methods. Our camera comes with a maximum shutter speed of $1 / 100,000$ second. We were able to modify the circuitry and reduce the shutter to $1 / 400,000$ second reducing the background by a factor of 13000. An interference filter of $25 \mathrm{~nm}$ bandwidth was placed between the lens and the CCD sensor. It reduces the background by another factor of 40 and together with the faster shutter increases the $\mathrm{S} / \mathrm{N}$ ratio to about 7 . This is sufficient in most cases. But when objects with high reflectivity (snow: $80 \%$, grass: $50 \%$ ) appear in the field-of-view while the laser projects the line on a low reflectivity target (e.g. asphalt: 5\%) the laser line is not the brightest return. It is possible to reduce the background by another factor of 2.5 by using a filter with a $10 \mathrm{~nm}$ bandwidth. But this narrow filter has a very restricted acceptance of angles and effectively narrows the aperture of the lens. It therefore reduces the signal strength at the same time it reduces $\mathrm{S} / \mathrm{N}$.

Background, which cannot be suppressed by a fast shutter or filter, is direct sunlight, specularly reflected sunlight, or direct light from lamps (e.g. rear lights of 
vehicles). Such light can be several orders of magnitude brighter and it can only be eliminated by background subtraction. We are planning to implement this in a future version of the system.

\section{Equipment}

We choose a camera with a high sensitivity in the near IR and a fast shutter. The GaAs laser electronics was built by ourselves. Usually the circuitry for a GaAs laser diode is designed to result in pulses of only a few ns. But in our case we wanted to get a large energy output and therefore we designed the laser to have pulses of about $1 \mu \mathrm{s}$. The vertical sync of the video output is used to trigger the laser, in this way we can use the laser with any camera with a NTSC output. Figure 2 shows the laser and the camera.

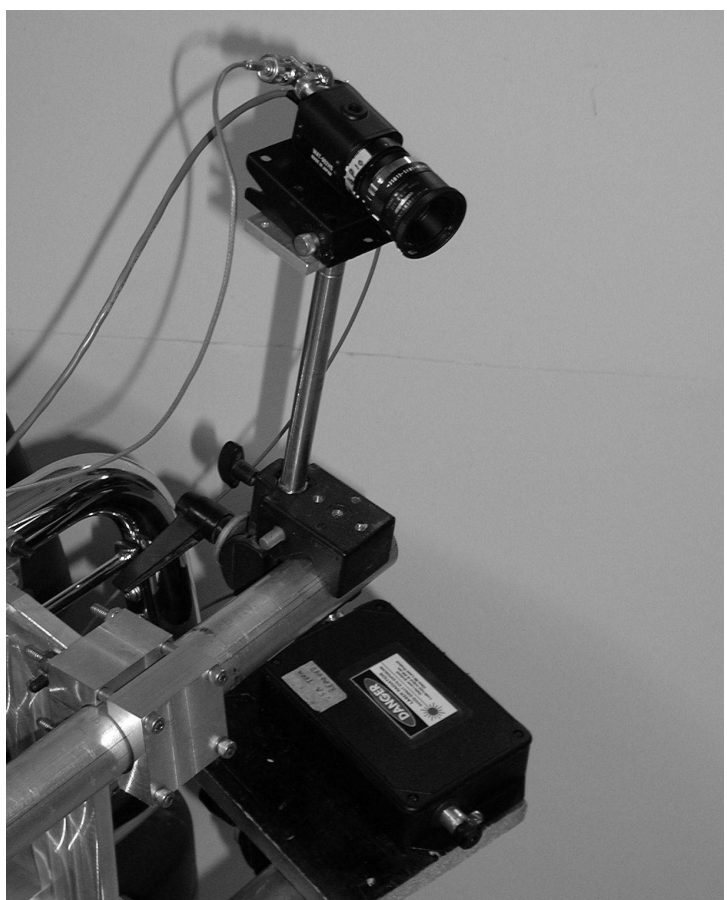

Figure 2 Laser and video camera in a typical configuration.

The video output is digitised with a frame grabber and analysed by a $\mathrm{C}++$ program. Any Pentium ${ }^{\circledR}$ II or faster $\mathrm{PC}$ processor allows the sensor to work in real-time (30 Hz). The parts for the laser, electronics, camera, and filter cost only a few hundred dollars.

\section{Results}

\section{Observing a cluttered environment}

In Figure 3 one can see objects visible to the camera of the laser line striper. In this case a large angle lens with a FOV of $105^{\circ}$ was mounted on the camera.

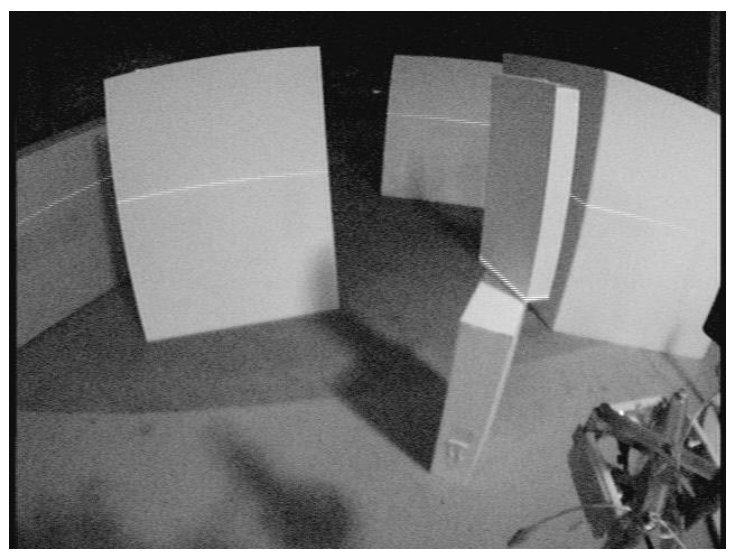

Figure 3 Scene observed by a laser line striper with a $105^{\circ}$ lens. The background is not suppressed in order to visualize the observed objects. The line going across the objects is the laser light.

The resulting distance measurements can be seen in Figure 4.

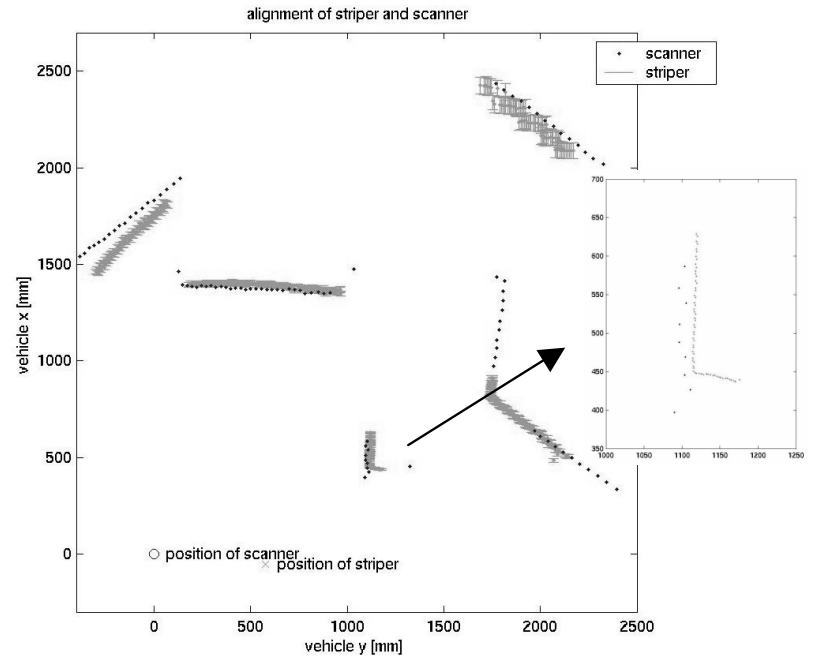

Figure 4 Distances of the scene seen in Figure 3 measured by the line striper (gray) and a laser scanner (black). Errors are shown for the striper points and are equivalent to $\mathbf{\pm 0 . 5}$ pixel in image coordinates. The insert shows a magnification of the box in front.

The largest distance measured in this scene is about $2.5 \mathrm{~m}$ and is the line at the top right hand corner of the 
Figure 4. One can notice that this line has discrete steps which are the result of the discrete nature of the locations in the image (pixels). The return from an object at this distance is too weak to achieve sub-pixel resolution. On the other hand sub-pixel resolution is possible for objects with strong return, like the box in front. In the insert of Figure 4 one can see the higher resolution of the line is which represents the box.

\section{Errors, limits, and failure modes}

The uncertainty in the distance is approximately the equivalent distance in world coordinates of one pixelto-pixel distance in the image. This equivalent distance is, like in all sensors using triangulation (e.g. stereo), approximately proportional to (see Figure 1 for definitions):

$$
\Delta d_{p} \sim d^{2} / s
$$

We tested the temporal stability of the measurements by comparing distance measurements from the same location but different times:

$$
\Delta d_{t}=\left|d\left(t_{1}\right)-d\left(t_{2}\right)\right|
$$

and found that on average

$$
\Delta d_{t} \approx 0.3 \cdot \Delta d_{p}
$$

A more global analysis of the errors can be done by comparing the measurements from the laser line striper with measurements from a different sensor. We used a $\mathrm{SICK}^{\circledR}$ laser scanner which has a resolution of $1 \mathrm{~cm}$ independent of distance. The comparison can be seen in Figure 4. The agreement is quite good. Disagreement is notable on the very left side and is due to incompletely corrected lens distortions at the edge of the image. Table 1 lists the ranges and resolutions for three camera lenses.

\begin{tabular}{l|c|c|c} 
field of view [deg] & 30 & 55 & 105 \\
\hline angular resolution [deg] & 0.05 & 0.09 & 0.16 \\
\hline maximum range [cm] & 700 & 520 & 300 \\
\hline range resolution [cm] & 1.4 & 2.6 & 5.0
\end{tabular}

Table 1 Ranges and resolutions for different camera lenses. The camera-laser separation $s=50$ cm.

The maximum range is the maximum distance at which a target with good reflectivity (paper) can still be seen. The range resolution is the "one-pixel distance" at a range of $2 \mathrm{~m}$.

There are two failure modes, false positives and false negatives. As mentioned in an earlier section, direct sunlight or light from a lamp (e.g. tail lights of another vehicle) produce background which can currently not be suppressed and they would appear as false positives. If one eliminates this background by background subtraction the sensor would most likely have a blind spot at this location, because such light usually saturates the camera. False negatives are caused by objects with very low Lambertian reflectivity or by objects occluding the view of the camera.

\section{Curb detection}

An important feature of a vehicle's surrounding is the curb. Its location signifies the boundary between vehicles and pedestrians in normal traffic situations. The laser line striper is well suited to measure the distance from the vehicle to the curb.

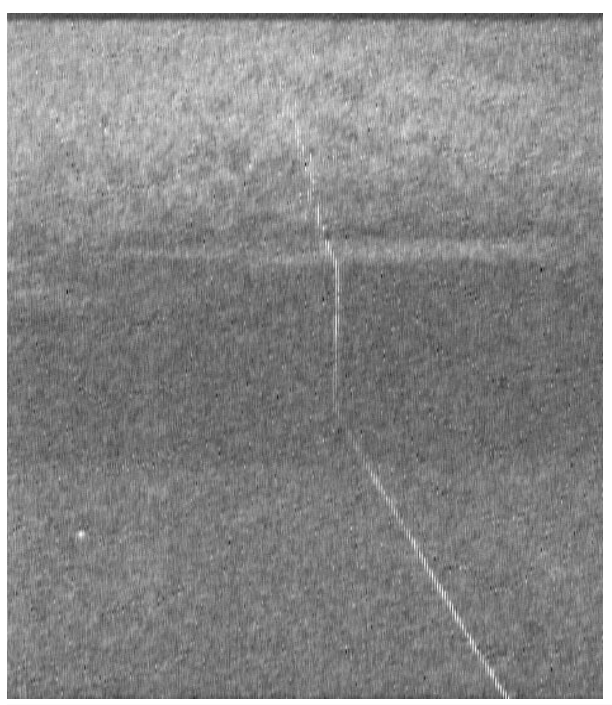

Figure 5 In the upper image the laser light illuminates the pavement, the curb, and the grass. The image was taken in bright sunlight. The lower pictures shows the output of the laser line striper with the vertical scale lines indicating distances of $10 \mathrm{~cm}$.

Figure 5 shows as an example of curb detection in bright sunlight. It is evident that the laser line striper can see the pavement, the curb, and the grass.

In the Fall of 2001 we installed a curb detector inside the front bumper of a transit bus and tested it during normal bus operation. We were able to show, that it is able to reliably detect and locate the curb. 


\section{Conclusion}

We have shown that the laser line striper is a costeffective range finder with resolutions of much less than $1^{\circ}$ in angles and around $1 \mathrm{~cm}$ in distance and a range of a few meters. The sensor can be configured to have a wide variety of angular coverage. It is eye-safe when it is appropriately packaged.

A further improvement will be to implement background subtraction which would eliminate direct light from the sun or lamps.

We intend to investigate more geometric configurations like increasing coverage to more than $180^{\circ}$ and using multiple lasers, fired at different times and at different angles to allow 3D coverage.

\section{Acknowledgements}

This work was partially sponsored by PennDOT under agreement \# 62N111, project TA-34, funded by the USDOT Federal Transit Administration, by the Robert Bosch Corporation, by by the Army Research Labs Collaborative Technology Alliance (CTA) Program, contract \# D AAD 19-01-2-0012, and by DARPA, contract \# MDA972-01-9-0015. The views and conclusions expressed are those of the authors and not necessarily those of the sponsors.

\section{References}

[1] Thorpe, C., Clatz, O., Duggins, D., Gowdy, J., MacLachlan, R., Miller, J.R., Mertz, C., Siegel, M., Wang, C., and Yata, T., Dependable Perception for Robots. Proceedings of International Advanced Robotics Programme IEEE, Robotics and Automation Society, Seoul, Korea, May, 2001.

[2] Mertz, C., McNeil, S., and Thorpe, C., Side Collision Warning Systems for Transit Buses. IV 2000, IEEE Intelligent Vehicle Symposium, October, 2000.

[3] Santos Conde, J.E., Hillebrand, M., Teuner, A., Stevanovic, N., Iurgel, U.; Hosticka, B.J., A smart airbag solution based on a high speed CMOS camera system. International Conference on Image Processing, 1999. ICIP 99, pp. 930 934 vol.3.

[4] Bursanescu, L., Bursanescu, M., Three-line highpower three-dimensional sensor, Proceedings of the SPIE - The International Society for Optical Engineering, vol.3313 p. 105-114.

[5] Yoshida, K., Hirose, S., Laser triangulation range finder available under direct sunlight, Proceedings of International Conference on Robotics and Automation, 1988, 1988 IEEE, pp. 1702 - 1708 vol.3.
[6] Matthies, L., Balch, T., Wilcox, B., Fast optical hazard detection for planetary rovers using multiple spot laser triangulation, Proceedings of the 1997 IEEE International Conference on Robotics and Automation, ICRA.

[7] American National Standards Institute, Inc., American National Standard for the Safe Use of Lasers, Z136.1-1993, The Laser Institute of America, 1993. (ANSI Z136.1-1993), p. $31,32,41,42$

[8] Camera Calibration Toolbox for Matlab ${ }^{\circledR}$, http://www.vision.caltech.edu/bouguetj/calib_do c/index.html .

[9] Zhao, J., Hao, J., Li, E., Guo, W., Yang, D., Method of improving CCD's resolution in laser triangulation measurement; Guangzi Xuebao/Acta Photonica Sinica, v26, n11, Nov, 1997, p 998-1002. 